\title{
Mathematical Techniques for Linear Wave-Structure Interactions
}

\author{
D.V. Evans \\ School of Mathematics, University of Bristol, Bristol, BS8 1TW, UK.
}

\section{Introduction}

It is difficult not to be impressed by the sheer range of Touviah Miloh's research output, and it is all the more remarkable that he has found the time to become a regular contributor to the Water Wave Workshops over many years. Each of his contributions is characterized by the application of powerful mathematical techniques coupled with a keen sense of the underlying physics of the problem under consideration. In the spirit of this approach, this paper offers a review of some of the mathematical techniques which have been employed to tackle a variety of classical linear waterwave problems involving fixed or floating structures, and which have provided analytical or semi-analytical solutions capable of useful physical interpretation. It is appropriate at this point to refer to the excellent monograph on the same topic by Chris Linton and Phil McIver (2001) and there is inevitably some overlap in choice of problems and techniques. My choice is a personal one reflecting my own interests and I would like to apologize in advance to those colleagues whose work is not mentioned in what follows which is primarily based on contributions from descendants of the Ursell school which have been so prominent at these Workshops from their inception.

We shall primarily be concerned with the effect of a plane wave incident upon a fixed or floating structure and we shall be using the classical linearized equations for water waves. This implies the existence of a velocity potential $\Phi(x, y, z, t)$ in the fluid region $0<y<h$, $-\infty<x, z<\infty$ excluding any bodies, in terms of Cartesian coordinates with $y$ measured vertically downwards in keeping with the Ursell notation. We shall be concerned with possible time harmonic solutions of radian frequency $\omega$ so that we seek a solution $\phi(x, y, z)$ where

$$
\begin{gathered}
\Phi(x, y, z, t)=\Re\left\{\phi(x, y, z) \mathrm{e}^{-i \omega t}\right\} . \\
\nabla^{2} \phi=0, \quad 0<y<h,-\infty<x, z<\infty, \\
\phi_{y}=0, \quad y=h,-\infty<x, z<\infty, \\
K \phi+\phi_{y}=0, \quad y=0,
\end{gathered}
$$

where $K=\omega^{2} / g$. On the surface of a rigid body in the fluid we have

$$
\phi_{n}=0 .
$$

In most of the cases that we shall consider the body will be a cylinder of infinite extent in the $z$ direction so that for an incident plane wave having crests parallel to the cylinder, the $z$ variation in the solution is constant and may be ignored. A further simplification will be the assumption of infinite depth of water. It now follows that

$$
\begin{gathered}
\phi \sim \phi_{0}\left(e^{-i K x-K y}+R e^{i K x-K y}\right), \quad x \rightarrow+\infty \\
\phi \sim \phi_{0} T e^{-i K x-K y}, \quad x \rightarrow-\infty
\end{gathered}
$$

The solution of these equations for one or more bodies of arbitrary shape, either fixed or making small timeharmonic oscillations, can always be treated numerically in both two or three dimensions, in finite depth or deep water, by integral equation methods using an appropriate Green function, and powerful commercial programmes exist for just this purpose. Much space in these Workshops has been used, and rightly so, to improve and assess these numerical schemes in response to commercial need as much as intellectual challenge. I shall not consider this body of work here but will concentrate instead on some of the ingenious mathematical techniques which have been employed to extract explicit solutions to the governing equations for simple geometries, or which have produced accurate approximate solutions thereby providing a deeper understanding of the problems considered.

\section{Reduction Methods and Complex Function Theory}

The first non-trivial result for vertical boundaries is the classical explicit result of Havelock (1929) who determined the potential everywhere in $x>0$ due to a given small time-harmonic oscillation of the boundary $x=0$. His explicit solution is

$$
\begin{array}{r}
\phi(x, y)=-\int_{0}^{\infty} F(k) \frac{K \sin k y-k \cos k y}{k\left(K^{2}+k^{2}\right)} e^{-k x} d k \\
-2 \int_{0}^{\infty} U(y) e^{-K y} d y
\end{array}
$$

satisfying

$$
\phi_{x}=U(y), \quad x=0
$$

where $U(y)$ is given and with $F(k)$ given by

$$
F(k)=2 \pi^{-1} \int_{0}^{\infty} U(y)(K \sin k y-k \cos k y) d y
$$


Although not entirely clear from his paper, Havelock implicitly uses the reduction method since his method is equivalent to introducing $\Phi$ defined by

$$
\Psi=K \phi+\phi_{y}
$$

to reduce the problem to one solvable for $\Psi$ by Fourier Sine Transforms and then integrating (11) to recover $\phi$.

The case of a barrier extending a finite distance $a$ into the fluid is much more difficult but Ursell (1947) utilized the Havelock solution above to obtain an explicit expression for the potential everywhere in the fluid and to determine $R$ as an explicit function of $K a$. In the course of his analysis he made use of the reduction method in applying the operator $K+\partial / \partial y$ to simplify a certain integral equation which arose in the analysis. His full explicit solution satisfying $\partial \phi / \partial x=0$ on $x=0,0<y<a$, is

$$
\phi=\phi_{0} e^{-i K x-K y}+\psi(x, y)
$$

where

$$
\phi_{0}=\pi I_{1}(K a)+i K_{1}(K a)
$$

and

$$
\begin{array}{r}
\psi(x, y)=\pi I_{1}(K a) e^{i K x-K y}+ \\
\int_{0}^{\infty} \frac{k \cos k y-K \sin k y}{k^{2}+K^{2}} J_{1}(k a) e^{-k x} d k
\end{array}
$$

for $x>0$ where $\psi(-x, y)=-\psi(x, y)$ determines the solution in $x<0$. Here $J_{1}, I_{1}, K_{1}$ are Bessel functions. It follows that $R=\pi I_{1}(K a) /\left(\pi I_{1}(K a)+K_{1}(K a)\right)$.

The reason that the reduction method works for vertical barrier problems follows from considering a complex-potential formulation of two-dimensional problems in deep water where $\phi$ is the real part of an analytic function $w(z)$ of the complex variable $z=$ $x+i y$. Thus the function $W(z)=d w / d z-i K w$ is real on $y=0$, its real part is constant on vertical fixed boundaries and it vanishes for large $|z|$. Thus for the Ursell problem it is easily shown that $W(z)=$ $z /\left(z^{2}+a^{2}\right)^{-1 / 2}-1$ and $w$ and hence $\phi$ are recovered by integration and application of conditions at infinity. $W$ can be found for any arrangement of vertical barriers but the details soon become complicated. See for example, Evans (1970), Porter (1974) and Mei (1966).

In finite depth, complex variable methods do not provide explicit solutions, but there is one notable exception due to Roseau who was concerned with determining $R$ and $T$ for the case of a wave incident in finite water depth over a bottom topography varying monotonically from one constant value at one infinity to a different one at the other infinity. He first transformed the original domain to an infinite strip at the cost of an $x$-dependence showing up in the wavenumber $K$ in the free-surface condition. He then showed that the new boundary-value problem in the uniform strip could be solved explicitly for a particular wide class of topographies. The success of his method depends on being able to reduce the problem to the solution of a two-term linear functional difference equation which allows an explicit solution. Roseau's paper, which was published in
French in 1960 but can be found in English translation in his book (1976), is a real tour de force and remains the only explicit solution for the propagation of waves over a variable bottom. Earlier work at the Courant Institute by John, Peters and others, used both reduction methods and complex analysis to obtain functional difference equations for a variety of uniform sloping beach problems, and references can be found in the book by Stoker (1957) and the major review article by Wehausen \& Laitone (1960). Further major simplifications of solutions to sloping beach problems can be found in a series of papers by Ehrenmark. (See for example 1998)

\section{Complementary Variational and Galerkin Methods}

The assumption of oblique incidence for infinitely long cylinders leads to a problem for a potential $\phi(x, y)$ satisfying the modified Helmholtz equation

$$
\phi_{x x}+\phi_{y y}-k^{2} \phi=0
$$

which pre-empts the use of analytic function theory. A useful real variable approach to problems involving oblique waves incident upon fixed rectangular boundaries in finite water depth is to match eigenfunction expansions in different regions across the common interval containing a vertical boundary. Thus for the Ursell barrier problem this can be done in two different ways providing singular integral equations for either the unknown horizontal velocity $u$ of the fluid under the barrier, or the jump in potential $p$ across it. In operator notation we have $K u=\psi(y), y \in \mathrm{E}_{g}$ and $(u, \psi)=A$, or $M p=\psi(y), y \in \mathrm{E}_{b}$ with $(p, \psi)=A^{-1}$. Here $K$ and $M$ are linear integral operators and $A$ is a real constant related to the complex reflection coefficient $R$ for the problem. A Galerkin approximation $\bar{u}$ to $u$ or $\bar{p}$ to $p$ provides lower and upper bounds for $A$ such that

$$
A_{l}=(\bar{u}, \psi) \leq A \leq(\bar{p}, \psi)=A_{u}
$$

The success of these complementary approximations depends on a good choice of trial functions in the Galerkin approximation. Porter and Evans (1995) were able to achieve high accuracy using a small number of expansion terms for a variety of barrier problems by choosing functions which reflected the physical behaviour at the ends of the barriers.In deep water Evans \& Morris (1972) ,used a one-term approximation consisting of the Ursell explicit solution for normallyincident waves on a single barrier to obtain accurate complementary bounds on the reflection coefficient for an obliqely-incident wave on a single barrier or on a pair of parallel vertical barriers. This latter problem in normally-incident waves has been solved analytically by Levine and Rodemich (1958) but the solution is too complicated to be of much use. In contrast the complementary variational approach enables us to show that a pair of vertical barriers is capable of completely reflecting an incident wave at certain frequencies and spacing, a result confirmed later by Newman (1974) using 
matched asymptotic expansions for closely-spaced barriers. This result led to the speculation that two pairs of such barriers, sufficiently widely spaced could support a trapped wave between them, providing an example of non-uniqueness of the two-dimensional water wave problem. Thirty years on this was confirmed using the accurate Galerkin method by Kuznetsov et al (2001) and extended to three dimensions by Shipway and Evans (2003) who showed numerically that a wave motion could be trapped between a pair of partially immersed concentric thin shells. See Section (6.)

\section{Multipole Methods and Wave-free Potentials}

We are indebted to Fritz Ursell for introducing a powerful new technique over fifty years ago for solving problems of radiation and scattering of small amplitude water waves by bodies of circular cross-section which has been exploited in a wide range of related problems since that time. In his third published paper (Ursell 1949) he considered the waves radiated by the heaving of a two-dimensional half-immersed oscillating circular cylinder centred at the origin. He constructed the solution by expanding in terms of a set of harmonic wave-free potentials

$$
\Phi_{m}(x, y)=\frac{\cos 2 m \theta}{r^{2 m}}+\frac{K \cos (2 m-1) \theta}{(2 m-1) r^{2 m-1}}
$$

each of which satisfied the free surface condition (4) were symmetric about $x=0$ and which vanished at large distances. To achieve a wave field at infinity he added to the expansion a wave source at the origin and by applying the final condition $\phi_{r}=U \cos \theta$ on $r=a$ he obtained an infinite system of equations for the unknown coefficients in the expansion which he showed was convergent. An alternative approach, which he adopted in a later paper on the fully submerged circular cylinder would have been to construct a set of multipoles or higher-order singularities at the origin, since it is easy to show that a symmetric multipole of order $n$ can be reduced to a combination of wave-free potentials and a wave source. The many subsequent approaches to radiation and scattering problems by circular cylinders have chosen to use expansions in terms of multipoles. The use of both symmetric and antisymmetric multipoles to solve for the scattering of a plane wave by a submerged circular cylinder led Ursell(1950) to a remarkable result, namely that the reflection coefficient $R$ was identically zero for all incident wave frequencies, all cylinder radii and all depths of submergence. The phenomenon of zero reflection is not new; it arises in the scattering by any pair of surface obstacles including thin barriers (Evans and Morris,1972) at discrete frequencies and geometries. But only for the circular cylinder in deep water is it zero for all values of two independent parameters. The surprises do not end there. Ogilvie (1963) showed that the waves radiated by such a cylinder in unit heave or sway motion were equal in amplitude and $\pi / 2$ out of phase from which it follows that a submerged cylinder making small circular motions radiates waves in one direction only along the free surface. A time-reversal argument now shows that it is possible for such a cylinder to completely absorb all the incident wave energy in a plane wave. The conditions under which this occurs were determined and confirmed experimentally by Evans et al (1979) and formed the basis for the Bristol Cylinder wave energy device.(Clare et al 1982)

Multipole potentials have also been used to solve radiation and scattering problems for a bottom -mounted vertical circular cylinder on the centre-plane of a narrow long wave tank. The governing equation is now the Helmholtz equation in two dimensions once the depth dependence has been extracted and the equations are the same as for an acoustic problem with a 'hard' condition on the side walls. The fundamental singularity of order $n$ for the Helmholtz equation is $H_{n}^{(1)}(k r) \mathrm{e}^{i n \theta}$. We can modify this free-space potential to correct for the Neumann conditions on the walls to obtain multipole potentials which can be used as expansion functions for either radiation or scattering problems for cylinders in narrow tanks. Such an approach is superior to a method based on successive images in the walls where any truncation scheme will fail to model the correct plane wave field at sufficiently large distances down the tank. In the multipole method, for $k d<\pi / 2$, where $2 d$ is the tank width, each multipole describes a plane wave at large distances. For $k d>\pi / 2$, all multipoles vanish down the tank and Callan et al(1992)were able to prove that in this case there existed a trapped mode having a unique wavenumber $k d$ for small $a / d$ where $a$ is the cylinder radius, and showed numerically that such a mode existed for all $a / d \leq 1$. This discovery played a key role in understanding the large exciting forces on cylinders near the centre of a large linear array of identical cylinders predicted using a powerful numerical code, by Maniar \& Newman (1997).

\section{Wiener-Hopf and Residue Calculus techniques}

The Wiener-Hopf technique is an ingenious method of obtaining explicit analytic solutions to integral equations with convolution-type kernels on a semi-infinite range, or boundary-value problems in a uniform strip or half-plane in which, on one boundary, different conditions are to be satisfied on each half-line. The classical prototype problem is that first solved by Sommerfeld for the scattering of a two-dimensional acoustic wave by a rigid half-plane. In the water wave context it has been used to determine the scattering of waves by a flexible ice sheet (Evans \& Davies,1969), a problem which was re-visited recently by Balmforth \& Craster(1999), Tkacheva (2001), and Chung \& Fox (2002). Again, Evans (1972), extending the work of Heins (1950), showed how the method could be used to construct a source function in a strip containing a submerged plane, thereby providing the fundamental tool needed for a numerical treatment of the scattering of waves by a change in bottom topography from one 
constant value to another different value.

Less well-known is the residue calculus method first given prominence in an electromagnetic context by Mittra \& Lee (1971) It also provides explicit solutions to mixed boundary-value problems in a strip using an entirely different approach from the Wiener-Hopf technique. Here, matching of eigen-function expansions in each half strip results in a first-kind infinite system of equations which can be expressed as the residue contributions from some of the poles of the integrand of a certain complex meromorphic function integrated around a sequence of increasingly large contours. Unlike the Wiener-Hopf technique, the method cannot handle half-plane problems but is more suited to problems in which one of the boundary conditions applies over a finite interval when it is possible to obtain a rapidly convergent solution by perturbing about the semi-infinite solution. Thus Evans (1991) was able to prove the existence of a trapped mode which makes small antisymmetric oscillations about a thin rigid strip which is on the centreline and parallel to the sides of an acoustical wave guide or wave tank. More recently Linton (2001) used the method to obtain an accurate solution to the classical finite dock problem in oblique waves whilst Chung \& Linton (2003) used the method to determine the scattering of flexural-gravity waves by a gap between two semi-infinite thin elastic sheets.

\section{Uniqueness Proofs}

Despite the apparent simplicity of the governing equations (1) to (7), until very recently little was known as to the circumstances under which any solutions of these equations were unique. Uniqueness is equivalent to replacing the conditions (6) and (7) by $\phi \rightarrow 0$ sufficiently rapidly as $x \rightarrow \pm \infty$. Much more is known when (2) is replaced by (15) for long cylinders, and Stokes (1846) showed that $\phi=e^{-k(x \cos \alpha+y \sin \alpha)}$ was a solution to these homogeneous equations over a uniform sloping beach of angle $\alpha$ provided $K=$ $k \sin \alpha$.These localized edge waves were generalized by Ursell (1952) who constructed a complicated combination of exponentials as a solution which showed that there were in fact $n$ bounded edge wave frequencies where $n$ satisfied $K=k \sin (2 n+1) \alpha$. A constructive derivation which includes unbounded solutions at the shore is due to Roseau (1958). Ursell (1951) also showed by constructing appropriate multipoles, that if $K<k$, there existed a non-uniqueness in the form of a trapping mode confined to the vicinity of a long submerged cylinder describing waves travelling along the cylinder and decaying in a direction normal to the cylinder axis. The existence of such modes over a general symmetric change in bottom topography was proved by Jones (1953)in a difficult paper using functional analysis.For surface obstacles in either two or three dimensions, most approaches have exploited the ideas of John (1950) using energy bounds to extend the conditions for uniqueness. John's proof is extremely simple. It is easy to show that

$$
\int_{W}|\nabla \phi|^{2} d x d y=K \int_{F}|\phi|^{2} d x .
$$

where $W$ is the water region and $F$ is the free surface excluding any partly-immersed bodies. From (4) and Havelock's result (8) we have

$$
\int_{0}^{\infty} \phi(x, y) e^{-K y} d y=0
$$

in deep water where there are no bodies for $x>0$. Integration by parts and use of the Schwarz inequality gives

$$
\begin{aligned}
K|\phi|^{2}(x, 0) & \leq K \int_{0}^{\infty}\left|\phi_{y}\right|^{2} d y \int_{0}^{\infty} e^{-2 K y} d y \\
& \leq \frac{1}{2} \int_{0}^{\infty}|\nabla \phi|^{2}(x, y) d y
\end{aligned}
$$

Integration over $x$ now gives an inequality which contradicts (18) unless $\phi=0$. Thus John concluded that the water wave problem was unique for a single partly-immersed body provided that its horizontal cross-section for $y>0$ did not exceed its surface value. Extensions of John's result are discussed at length by Kuznetsov et al(2002).

Until recently the strictly two-dimensional problem for surface or submerged obstacles was presumed to be unique as it was not clear how a local oscillation could remain trapped by a partially submerged body or bodies. The question was finally resolved once and for all when McIver (1996) constructed a simple counterexample to show that the potential

$$
\phi=\int_{0}^{\infty} e^{-k y} \frac{\cos k(x-a)+\cos k(x+a)}{k-K} d k
$$

when $K a=\pi / 2$ described a local two-dimensional oscillation between a certain family of pairs of surfacepiercing cylindrical cross-sections. Since then numerous examples have been discovered in both two and three dimensions including cases of submerged pairs of bodies. It is still not known however whether a single surface-piercing body can exhibit a non-unique solution. For an up-to-date review of current uniqueness proofs, including trapped modes, see Kuznetsov et al (2002).

\section{Conclusion}

In this brief review I have touched upon some of the powerful mathematical techniques which have been introduced to solve the deceptively simple equations of linear water waves whose solutions continue to frustrate and delight successive generations of mathematicians.

\section{References}

N.J. Balmforth \& R.V. Craster, 1999, Ocean waves and ice sheets. J. Fluid. Mech, 395, 89-124. 
M. Callan, C.M. Linton \& D.V. Evans, 1991, Trapped modes in two-dimensional waveguides. $J$. Fluid. Mech, 229, 51-64.

H. Chung \& C. Fox, 2002, Calculation of wave-ice interaction using the Wiener-Hopf technique. New Zealand J. Maths. 31, 1-18.

H. Chung \& C.M. Linton, 2003, Interaction between water waves and elastic plates using the residue calculus technique. 18th Int. Workshop on Water Waves and Floating Bodies, Le Croisic, France.

R. Clare, D.V. Evans \& T.L. Shaw, 1982, Harnessing sea wave energy by a submerged cylinder device. $J$. Inst. Civil Eng., 73, 356-385.

D.V. Evans, 1970, Diffraction of water waves by a submerged vertical plate. J. Fluid Mech., 40, 433-451.

D.V. Evans, 1972, The application of a new source potential to the problem of the transmission of waves over a shelf of arbitrary profile. Proc. Camb. Phil. Soc., 71, 391-410.

D.V. Evans 1992, Trapped acoustic modes. J.Appl. Maths., 49, 45-60.

D.V. Evans \& T.V. DAviEs, 1968, Wave-ice interaction. Davidson Laboratory Rep. no.1313, Stevens Institute of Technology, NJ USA

D.V. Evans, D.C. Jeffrey, S.H. Salter \& J.R.M. TAYLOR, 1979, Submerged cylinder wave energy device: theory and experiment. Appl. Ocean Res., 1, 312.

D.V. Evans \& C.A.N. Morris, 1972, Complementary approximations to the solution of a problem in water waves. J. Inst. Maths. Applics., 10, 1-9.

U.T. EHrEnMARK,1998, Oblique wave incidence on a plane beach:the classical problem revisited. J. Fluid Mech., 368, 291-319.

T.H. Havelock, 1929, Forced surface-waves on water. Philosophical Magazine, viii, 569-576.

A.E. Heins, 1950, Water waves over a channel of finite depth with a submerged plane barrier. Can. J. Math., 2, 210-222.

F. John,1950, On the motion of floating bodies.II Comm. Pure Appl. Math. 3, 45-101.

D.S. Jones 1953, The eigenvalues of $\nabla^{2} u+\lambda u=0$ when the boundary conditions are given in semi-infinite domains. Proc. Camb. Phil. Soc., 49, 668-684.

N. Kuznetsov, P. McIver \& C.M. Linton, 2001, On uniqueness and trapped modes in the water-wave problem for vertical barriers. Wave Motion, 33, 283307.

N. Kuznetsov, V. Maz'YA \& B.VAinBerG, 2002, Linear Water Waves. A Mathematical Approach Cambridge University Press

H. Levine \& E. Rodemich, 1958, Scatteri ng of surface waves on an ideal fluid. Math. and Stat. Lab Tech. Report No. 78, Stanford University.

C.M.Linton, 2001, The finite dock problem. ZAMP, 52, 640-656.
C.M. Linton \& P. McIVer, 2001, Handbook of Mathematical Techniques for Wave/Structure Interactions. Chapman \& Hall/CRC.

H.D. Maniar \& J.N. NeWman, 1997, Wave diffraction by a long array of cylinders. J. Fluid. Mech, 339, 309-330.

M. MCIver, 1996, An example of non-uniqeness in the two-dimensional linear water wave problem. J. Fluid. Mech, 315, 257-266.

C.C. MEI, 1966, Radiation and scattering of transient gravity waves by vertical plates. Quart. J. Mech. Appl. Math., 19, 417-440.

R. Mittra \& S.W. LEE, 1971, Analytic techniques in the theory of guided waves. New York: Macmillan.

J.N. NEWMAN, 1974, Interaction of water waves with two closely-spaced barriers. J.Fluid Mech, 66, 97-106.

T.F. Ogilvie, 1963, First-and second-order force on a cylinder submerged under a free surface. J. Fluid Mech, 16, 451-472.

D. Porter, 1974, The radiation and scattering of surface waves by vertical barriers. J. Fluid Mech., 63, 625-634.

R. Porter \& D.V. Evans,1995, Complementary approximations to wave scattering by vertical barriers. $J$. Fluid Mech, 294, 155-180.

M. Roseau, 1976, Asymptotic Wave Theory Amsterdam: North-Holland.

M. Roseau, 1958, Short waves parallel to the shore over a sloping beach. Comm. Pure Appl. Math. 11, 433-493.

B.J. Shipway \& D.V. Evans, 2003, Wave trapping by axisymmetric concentric cylinders. J. Offshore Mech. Arct. Eng.,125(1), 59-64.

G.G.STOKES, 1846, Report on recent researches in hydrodynamics. Rep. 16th Brit. Ass. Adv Sci., London $1-20$.

J.J.StokeR, 1957, Water Waves New York: Interscience

F. URSELL, 1947, The effect of a fixed vertical barrier on surface waves in deep water. Proc. Camb. Phil. Soc., 42, 374-382.

F. URSELL, 1949, On the heaving motion of a circular cylinder on the surface of a fluid. Quart. J. Mech. Appl., 2, 218-231.

F. URSELl, 1950, Surface waves on deep water in the presence of a submerged circular cylinder I. Proc. Camb. Phil. Soc.,46, 141-152.

F. URSELL, 1951, Trapping modes in the theory of surface waves. Proc. Camb. Phil. Soc., 47, 347-358.

F. Ursell, 1952, Edge waves on a sloping beach. Proc. Roy. Soc. London, A214, 79-97.

J.V. Wehausen \& E.V. Laitone, 1960, Surface Waves Handbook of Physics, Springer, ix. 
